\title{
ADESÃO AO GRUPO DE CESSAÇÃO ENTRE TABAGISTAS DE UNIDADE BÁSICA DE SAÚDE
}

\author{
Antonio Augusto Claudio Pereira' , Lucas Johann Gritsch², Mariana Soares Passos ${ }^{1}$, Marcela \\ Demitto Furtado 3
}

\begin{abstract}
RESUMO: Objetivo: verificar o perfil de usuários tabagistas e compreender os aspectos relacionados à adesão ao grupo de cessação. Método: estudo transversal com tabagistas de uma Unidade Básica de Saúde de Maringá, estado do Paraná. A coleta e a análise descritiva dos dados ocorreram entre agosto e setembro de 2016. Resultados: participaram do estudo 72 tabagistas; 51 desconheciam o Programa Nacional de Controle do Tabagismo e a proposta de grupos de cessação. As barreiras identificadas para a não adesão aos grupos foram dificuldade de locomoção à unidade de saúde e incompatibilidade de horários com a rotina dos tabagistas. Entre os entrevistados, 42 mostraram interesse em participar de intervenção voltada ao controle do tabagismo, sugerindo maior acesso a informações e mudanças na abordagem das atividades. Conclusão: há a necessidade de valorizara busca ativa dos indivíduos na comunidade e abordar dinamicamente os grupos para atender a real necessidade dos participantes. As variáveis levantadas podem contribuir para maior participação de tabagistas no programa e para a cessação do hábito de fumar.

DESCRITORES: Atenção primária à saúde; Abandono do hábito de fumar; Programa nacional de controle do tabagismo; Tabaco; Saúde pública.
\end{abstract}

\section{ADHERENCE TO A SMOKING CESSATION GROUP BY SMOKERS ASSISTED AT A BASIC HEALTH UNIT}

ABSTRACT: Objective: to analyze the profile of smokers assisted at a basic health unit and understand the aspects related to adherence to a smoking cessation group. Method: Cross-sectional study with smokers assisted at a Basic Health Unit in Maringá in the State of Paraná. Data collection and descriptive analysis were performed in August-September 2016. Results: Seventy-two (72) smokers participated in the study. Of these, 51 were unaware of the National Tobacco Control Program (PNCT) and the proposed cessation groups. The barriers to adherence to the intervention groups identified in this study were difficult access (transportation) to the health unit and inability to join the groups due to the smokers' routine. Forty-two (42) respondents were willing to participate in a group intervention targeted to tobacco control and said that greater access to information and changes in the approaches used in these interventions were necessary. Conclusion: active search of the individuals in the community and use of a dynamic approach in group interventions are needed to meet the real needs of the participants. The variables identified may contribute to increase the participation of smokers in the program and, hence, smoking cessation rates.

KEYWORDS: Primary health care; Smoking cessation; National tobacco control program; Tobacco; Public health.

\section{ADHESIÓN AL GRUPO DE ABANDONO ENTRE FUMADORES EN UNIDAD BÁSICA DE SALUD}

RESUMEN: Objetivo: Verificar perfil de fumadores, comprender aspectos relativos a adhesión al grupo de abandono. Método: Estudio transversal con fumadores de Unidad Básica de Salud de Maringá, Paraná. Datos recolectados y analizados descriptivamente de agosto a setiembre de 2016. Resultados: Participaron 72 fumadores; 51 desconocían el Programa Nacional de Control del Tabaquismo y la propuesta de grupos de abandono. Se identificaron como barreras para la no adhesión: dificultad de traslado a la unidad de salud e incompatibilidad horaria con rutinas de los fumadores. Cuarenta y dos entrevistados estuvieron interesados en participar de intervención orientada al control del tabaquismo, lo que sugiere mayor acceso a información y cambios de abordaje de las actividades. Conclusión: Es necesario valorar la búsqueda activa de los individuos de la comunidad y atender las necesidades reales de los participantes. Las variables relevadas favorecen mayor participación de fumadores en el programa y para abandonar el hábito de fumar.

DESCRIPTORES: Atención Primaria de Salud; Cese del Hábito de Fumar; Programa Nacional de Control del Tabaquismo; Tabaco; Salud Pública.

1Acadêmico do Curso de Medicina do Centro Universitário Cesumar. Maringá, PR, Brasil.
${ }^{2}$ Acadêmico do Curso de Medicina da Universidade Positivo. Curitiba, PR, Brasil.
${ }^{3}$ Enfermeira. Doutora em Enfermagem. Docente do Departamento de Medicina do Centro Universitário Cesumar. Maringá, PR, Brasil. 


\section{INTRODUÇÃO}

Estima-se que um terço da população mundial adulta seja fumante, o que caracteriza o tabagismo como doença epidêmica. Esse hábito configura-se como a principal causa de mortes evitáveis, relacionando-se ao óbito prematuro de aproximadamente seis milhões de indivíduos, principalmente em países subdesenvolvidos ou em desenvolvimento ${ }^{(1)}$.

Um dos principais fatores de risco para as doenças crônicas não transmissíveis, o fumo é responsável por $71 \%$ dos casos de câncer de pulmão, $42 \%$ das doenças respiratórias crônicas e $10 \%$ das doenças cardiovasculares, gerando suntuosos gastos com internações, medicamentos e diminuição de produtividade(2).

Pesquisa da Organização Mundial da Saúde revela que, se as tendências de uso do tabaco forem mantidas, em 2030, ocorrerão mais de oito milhões de mortes, 80\% destas em países de baixa e média renda ${ }^{(3)}$.

No Brasil, estima-se que 156.200 pessoas morram a cada ano por doenças associadas ao fumo ativo e passivo, mortes essas que se concentram em populações mais vulneráveis ${ }^{(4)}$. Acredita-se que o custo com as consequências do consumo de produtos derivados do tabaco no país seja de quase $\mathrm{R} \$ 57$ milhões por ano, sendo 39,3 bilhões por assistência médica e tratamento e 17,5 bilhões por perda de produtividade $^{(5)}$.

Diante desse cenário, o Ministério da Saúde, juntamente com o Instituto Nacional de Câncer (INCA), lançou em 1986 o Programa Nacional de Controle do Tabagismo (PNCT), cujos objetivos estratégicos estão fundamentados em três pilares: (i) prevenção da iniciação, (ii) promoção da cessação e (iii) proteção ao tabagismo passivo/ambientes livres-abertos ${ }^{(6-7)}$. Em 2013 foi publicada a Portaria n. 0571 do Ministério da Saúde, modernizando as diretrizes do cuidado ao indivíduo tabagista no âmbito da rede de atenção à saúde das pessoas com doenças crônicas do Sistema Único de Saúde (SUS) ${ }^{(8)}$.

O auxílio à cessação tabágica inclui o aconselhamento comportamental, que pode ou não estar associado ao tratamento farmacológico ${ }^{(9)}$. As intervenções grupais, pela boa relação custo-benefício, permitem assistência simultânea a múltiplas pessoas, possibilitando que o indivíduo sinta-se acolhido ${ }^{(10)}$. Dessa forma, acredita-se que a Atenção Primária à Saúde (APS) consiga exercer impacto positivo no controle do tabagismo, por se tratar de um cenário que aproxima os profissionais da saúde e a comunidade, com foco em ações de promoção, manutenção e melhora da saúde ${ }^{(11)}$.

O PNCT permitiu ao Brasil estar entre os países cujas políticas de controle de tabaco encontramse em estágios avançados ${ }^{(12)}$. As diversas ações do Programa são inovadoras, no entanto, ainda são necessárias estratégias que reduzam de forma significativa a prevalência do tabagismo ${ }^{(13)}$.

Estudo realizado em uma universidade pública, por meio da implantação de um Programa de Cessação do Tabagismo (PCT), identificou que, dos 128 fumantes inscritos,76\% compareceram ao primeiro encontro, dos quais $71 \%$ efetivamente concluíram o PCT. Os grupos que realizaram avaliação de seguimento alcançaram taxas de abstinência de $27 \%$ a $32 \%$, superiores às esperadas em tratamento unicamente psicológico. Infere-se que estratégias de recrutamento que combinem estratégias ativas (contatos pessoais, convites personalizados, entrevistas individuais) e reativas (cartazes, e-mails corporativos, divulgações em jornais) sejam mais eficazes para captação e adesão de fumantes ${ }^{(10)}$.

O impacto relevante do tabagismo na saúde da população aponta a importância de superar limitações e obstáculos que dificultam a real implementação do PNCT na atenção básica, o que poderia aumentar o sucesso do programa de $3 \%$ a $30 \%$ em 1 ano $^{(1)}$. Nesse sentido, o presente estudo teve como objetivo verificar o perfil de usuários tabagistas de uma Unidade Básica de Saúde e compreender aspectos relacionados à adesão ao grupo de cessação. 


\section{MÉTODO}

Trata-se de estudo descritivo, transversal, desenvolvido com tabagistas pertencentes à área de abrangência de uma Unidade Básica de Saúde (UBS) do município de Maringá, Paraná.

Os participantes do estudo foram usuários tabagistas cadastrados na referida unidade. O critério de inclusão foi tabagistas residentes na área de abrangência da UBS de estudo, e o de exclusão, tabagistas com idade inferior a 18anos.

A população de tabagistas cadastrada na UBS era de 146 indivíduos, no entanto participaram da pesquisa 72, já que 66 não foram localizados e oito recusaram-se a participar.

A UBS do estudo oferece grupos de cessação tabágica, os quais iniciam suas atividades quando atingem um número de inscritos superior a 10. O grupo acontece semanalmente e as suas atividades são encerradas após um período de aproximadamente 2 meses.

A coleta de dados ocorreu entre os meses de agosto e setembro de 2016, por meio de entrevistas, as quais foram realizadas preferencialmente por telefone. Contudo, a dificuldade de contato telefônico, por conta de números inexistentes ou pelo não atendimento das chamadas, mesmo após três tentativas em horários diferentes, compeliu a realização de 46 entrevistas por meio de visita domiciliar. A duração média da realização das entrevistas, tanto por telefone quanto por meio da visita domiciliar, foi de 20 minutos.

Nas entrevistas foi utilizado um instrumento estruturado com 32 questões, elaborado pelos próprios autores e testado previamente com uma população não selecionada para o estudo. $\mathrm{O}$ instrumento apresentava as seguintes variáveis: a) sociodemográficas: idade, sexo, renda familiar, estado civil, escolaridade e cor da pele; b) relacionadas ao hábito de fumar: carga tabágica, ingestão de álcool e uso de drogas ilícitas, prática de exercício físico, motivação e autopercepção dos prejuízos; c) relacionadas ao conhecimento e à visão sobre os grupos de cessação: conhecimento da existência do PNCT e dos tratamentos, participação em grupos, fatores de adesão e não adesão e interesse em parar de fumar.

A organização, tabulação e sumarização dos dados foram realizadas no Microsoft Excel 2010. Depois dessa etapa, utilizou-se de análise descritiva para a interpretação dos dados.

A pesquisa foi aprovada pela Secretaria de Saúde do município do estudo e pelo Comitê de Ética em Pesquisa do Centro Universitário Cesumar, com parecer n.ํㅜ 1.694.692.

\section{RESUltados}

Participaram do presente estudo 72 tabagistas, dos quais 37 (51,39\%) eram do sexo feminino, 42 (58,33\%) com idade entre 35 e 59 anos, 52 (72,22\%) de cor branca, 33 (45,83\%) casados e 37 (51,39\%) com 8anos ou mais de estudo. Quanto à renda familiar mensal, 32(44,44\%) indivíduos recebiam entre $\mathrm{R} \$ 880,00$ e 1.760,00 (Tabela 1).

Tabela 1- Características sociodemográficas de tabagistas pertencentes a uma Unidade Básica de Saúde. Maringá, PR, Brasil, 2016. (continua)

\begin{tabular}{llc}
\hline Variáveis & $\mathbf{N}$ & $\%$ \\
\hline Sexo & & \\
$\quad$ Masculino & 35 & 48,61 \\
$\quad$ Feminino & 37 & 51,39 \\
\hline Idade & \multicolumn{2}{c}{} \\
$\quad 18$ a 34 & 9 & 12,50 \\
35 a 59 & 42 & 58,33 \\
$\geq 60$ & 21 & 29,17 \\
\hline
\end{tabular}




\begin{tabular}{lcc}
\hline Cor & & \\
Branca & 52 & 72,22 \\
Parda & 19 & 26,39 \\
Amarela & 1 & 1,39 \\
\hline Estado civil & & \\
$\quad$ Casado & 33 & 45,83 \\
Solteiro & 22 & 30,56 \\
Divorciado & 8 & 11,11 \\
Viúvo & 7 & 9,72 \\
Outros & 2 & 2,78 \\
\hline Tempo de estudo (anos) & & \\
$<8$ & 35 & 48,61 \\
$\geq 8$ & 37 & 51,39 \\
\hline Renda familiar & & \\
De $R \$ 880,00$ a $R \$ 1,760,00$ & 32 & 44,44 \\
De $R \$ 1,760,00$ a $R \$ 3,520,00$ & 22 & 30,56 \\
De $R \$ 3,521,00$ a $R \$ 5,280,00$ & 10 & 13,89 \\
$>R \$ 5,280,00$ & 1 & 9,38 \\
Ignorado & 7 & \\
\hline
\end{tabular}

Em relação às características relacionadas ao hábito de fumar, observou-se que $30(41,67 \%)$ tabagistas fumavam havia mais de 30 anos e 17(23,62\%) fumavam mais de 20 cigarros/dia. A ansiedade foi o principal estímulo para fumar, o que foi evidenciado pela resposta de $46(63,8 \%)$ tabagistas, e os outros motivos foram apontados pelos indivíduos como o próprio vício, o período pós-prandial, problemas familiares, cirurgia bariátrica, álcool e solidão. Quanto ao principal obstáculo para deixar o vício, 45 $(57,69 \%)$ referiram a falta de interesse, além de mencionarem a demora na abertura de novas turmas para o grupo de apoio, a abordagem pouco atrativa e o insucesso em tentativas anteriores. Destaca-se ainda que 51 (70,83\%) indivíduos já tentaram parar de fumar em algum momento da vida (Tabela 2).

Tabela 2 - Características relacionadas ao hábito de fumar entre tabagistas pertencentes a uma Unidade Básica de Saúde. Maringá, PR, Brasil, 2016. (continua)

\begin{tabular}{lcc}
\hline Variáveis & N & \% \\
\hline Tempo de consumo (anos) & 9 & 12,5 \\
$\leq 10$ anos & 14 & 19,44 \\
$11-20$ anos & 19 & 26,39 \\
$21-30$ anos & 30 & 41,67 \\
$>30$ anos & & \\
\hline Quantidade cigarros/dia (Unidade) & 31 & 43,05 \\
$\quad 10$ & 24 & 33,33 \\
$11-20$ & 17 & 23,62 \\
$>20$ & & \\
\hline Principais estímulos para fumar & 46 & 63,8 \\
$\quad$ Ansiedade & 30 & 41,6 \\
$\quad$ Situação de estresse & 37 & 51,38 \\
Café & 33 & 45,83 \\
$\quad$ Outros & & \\
\hline Principais obstáculos para parar de fumar & 45 & 57,69 \\
$\quad$ Falta de interesse & 9 & 11,54 \\
$\quad$ Falta de tempo & & \\
\hline
\end{tabular}




$\begin{array}{llr}\text { Falta de incentivo } & 8 & 10,26 \\ \text { Falta de informação } & 3 & 1,28 \\ \text { Problemas de locomoção até a Unidade Básica de Saúde } & 7 & 8,97 \\ \text { Outros } & 8 & \end{array}$

\begin{tabular}{llr}
\hline Já tentou parar de fumar & & \\
Sim & 51 & 70,83 \\
Não & 21 & 29,17 \\
\hline
\end{tabular}

Entre os participantes, 32 (44,44\%) faziam uso de bebidas alcoólicas frequentemente e $11(15,27 \%)$ já haviam usado drogas ilícitas ao menos uma vez na vida. Quando questionados sobre atividade física, apenas $24(33,33 \%)$ relataram praticar alguma modalidade.

Pouco mais da metade dos entrevistados, 37(51,39\%), reconheceu os prejuízos que o hábito trouxe à sua saúde, e as principais queixas foram: tosse matutina, relatada por 14 (19,44\%) indivíduos, 11 $(15,28 \%)$ referiram rouquidão, quatro $(5,56 \%)$ perda ponderal, $11(15,28 \%)$ dispneia e oito $(11,11 \%)$ nervosismo quando em abstinência.

Com relação à terapêutica adotada para o combate ao tabagismo, 42 (62\%) participantes disseram conhecer os tipos de tratamento disponíveis, embora a maioria não soubesse especificar quais eram, demonstrando desconhecimento inclusive quanto à terapia medicamentosa.

Quando questionados sobre a existência de um programa específico para auxiliar na cessação do vício - o PNCT -, 51(70,33\%) disseram nunca ter tido conhecimento. Sobre a participação nos grupos de cessação ou apoio psicocomportamental, 64 (88,89\%) afirmaram nunca ter participado. Os motivos para a não adesão a esses tipos de grupo foram dificuldade de locomoção até a UBS 47 (73,43\%), falta de informação sobre o programa $17(26,56 \%)$ e incompatibilidade com a rotina ou falta de tempo 11 $(17,18 \%)$ (Tabela 3).

Como sugestões para melhorar a adesão ao grupo, 31 (36,04\%) participantes consideraram ampliar as informações sobre o programa e seus resultados, 27 (31,39\%) apontaram mudanças na abordagem das atividades e 23 (26,75\%) mencionaram maior oferta de horários. Destaca-se que 42 (58,33\%) entrevistados manifestaram interesse em participar de uma intervenção voltada ao controle do tabagismo (Tabela 3).

Tabela 3 - Aspectos relacionados à adesão aos grupos de cessação por tabagistas pertencentes a uma Unidade Básica de Saúde. Maringá, PR, Brasil, 2016. (continua)

\begin{tabular}{lcc}
\hline Questões & $\mathbf{N}$ & $\%$ \\
\hline Participação em algum grupo de cessação ou apoio psicocomportamental & & \\
Não & 64 & 88,89 \\
Sim & 8 & 11,11 \\
\hline Ciência quanto à existência do PNCT & 51 & 70,33 \\
$\quad$ Não & 21 & 29,67 \\
Sim & 47 & \\
\hline Motivos de não adesão ao grupo & 17 & 73,43 \\
Dificuldade de locomoção até a UBS & 11 & 26,56 \\
Falta de informações & 31,18 \\
Horário incompatível com a rotina ou falta de tempo & 36,04 \\
\hline Sugestões para melhora da adesão ao grupo & 27 & 31,39 \\
Mais informações sobre o programa e seus resultados & 23 & 26,75 \\
Mudanças na abordagem das atividades & 5 & 5,82 \\
Mais horários disponíveis & & \\
Transporte gratuito & 42 & 58,33 \\
\hline Interesse em participar de uma intervenção voltada ao controle do tabagismo & 30 & 41,67 \\
Sim & & \\
Não & & \\
\hline
\end{tabular}




\section{DISCUSSÃO}

Apesar das tentativas e programas de cessação tabágica, o número de fumantes que participa das atividades oferecidas, em particular do grupo cognitivo-comportamental, ainda é bastante reduzido. Destaca-se que a dificuldade de locomoção até a unidade ou a incompatibilidade de horários ainda representam importantes obstáculos para o sucesso dos grupos, o que incita os benefícios potenciais da formação de grupos em horários alternativos.

A escassez de informações quanto à existência dos grupos na APS foi outra barreira identificada, e bastante relevante para a não adesão dos tabagistas à atividade, apontando a necessidade de melhorar os meios de divulgação das atividades, grupos e acessibilidade de informações. Estudo verificou o importante papel do recrutamento ativo, combinando novas tecnologias com estratégias mais individualizadas, de modo a personalizar as necessidades dos fumantes com a finalidade de contribuir para a maior adesão aos grupos ${ }^{(10)}$. Nesse sentido, é preciso capacitar os profissionais que trabalham com grupos, com o objetivo de explorar melhor os recursos disponíveis no SUS para garantir a acessibilidade de todos aos serviços ${ }^{(14)}$.

Ainda, muitos usuários não hesitam em manifestar falta de interesse. No ano de 2008, a Sociedade Brasileira de Pneumologia e Tisiologia apresentou diversas diretrizes para a cessação do tabagismo, entre elas a avaliação do grau de motivação do indivíduo, a fim de que os profissionais compreendam melhor o nível de prontidão para a mudança de hábito e assim conduzam suas ações com maior acolhimento, escuta e compreensão ${ }^{(15)}$.

Pesquisa realizada com o objetivo de investigar os motivos do abandono do tratamento no PNCT identificou relação com o local em que o programa é desenvolvido, o tipo de terapia utilizada, o horário das reuniões e os profissionais que prestaram a assistência ${ }^{(16)}$.

A utilização da terapia cognitivo-comportamental como única estratégia também parece não favorecer a continuidade do tratamento para a cessação tabágica ${ }^{(16)}$, o que suscita uma abordagem mais criativa e focada nas necessidades do outro, visando, assim, maior resolutividade. A utilização de técnicas integrativas à terapia comportamental, como o estímulo à criação de uma rede de apoio entre os participantes (p. ex.: trocas de experiências, criação de lista de contato telefônico, etc.), parece auxiliar não apenas durante o tratamento, mas também depois de seu término ${ }^{(17)}$.

Nesse sentido, diversos estudos enaltecem a importância da qualificação profissional como ação estratégica para a consolidação das intervenções antitabaco ${ }^{(10-11,18)}$. Faz-se necessário o trabalho de equipe motivada e capaz de oferecer alternativas que entusiasmem o fumante e contribuam para o processo de cessação e, ao mesmo tempo, preparada para lidar com eventuais insucessos.

Os tratamentos com grupos de cessação são positivos ao oferecerem suporte social - troca de experiências quanto às peculiaridades do comportamento tabágico e às dificuldades em deixá-lo. Contudo, reuniões individualizadas permitem maior especificidade em relação às necessidades e características do indivíduo que fuma ${ }^{(17)}$. Ainda, abordagens mais dinâmicas, direcionadas aos fatores que despertam o desejo de fumar, podem ter resultado positivo no estímulo do interesse pela participação, aumentando a adesão às atividades já estabelecidas.

A associação dessas abordagens com a terapia medicamentosa pode melhorar os índices de sucesso da cessação do tabagismo ${ }^{(19)}$, no entanto, conforme evidenciado neste estudo, essa forma de tratamento ainda é pouco difundida. Nesse sentido, faz-se necessário informar melhor os usuários sobre a possibilidade de associação farmacológica e tornar essa realidade mais próxima deles.

A ansiedade e as situações de estresse representam grandes alvos, passíveis de intervenções, uma vez que foram indicados como gatilhos importantes para o desejo de fumar. Assim, atividades com educador físico, laborais, técnicas de relaxamento ou terapia com antidepressivos podem exercer um papel significativo(20).

Do ponto de vista sociodemográfico, nacionalmente se sabe que o tabagismo é superior no sexo masculino, na raça/cor preta e na região rural. Em relação à escolaridade, a proporção de fumantes é 
inversamente proporcional aos anos de estudo. Além disso, quanto menor a renda, maior a prevalência de fumantes ${ }^{(21)}$. Tais dados divergem dos encontrados na presente pesquisa, o que pode ser justificado pelo fato de a UBS estar localizada numa área de maior poder aquisitivo, com população adstrita que apresenta características particulares.

No geral, uma parcela importante da população tabagista apresenta baixo nível de escolaridade, o que implica a adequação de estratégias de informação acerca dos malefícios do tabaco e de ferramentas de cessação disponíveis ${ }^{(22)}$.

Outro dado relevante encontrado, ao analisar o tempo de consumo do tabaco, é que a maioria dos indivíduos iniciou o hábito na adolescência. Alguns autores consideram o tabagismo uma doença pediátrica, principalmente nos países em desenvolvimento ${ }^{(16)}$. Jovens depressivos, na maioria das vezes, recorrem ao cigarro e desenvolvem intensa dependência à nicotina ${ }^{(23)}$. Logo, seria interessante $\mathrm{O}$ desenvolvimento de ações direcionadas à prevenção do início do tabagismo, especialmente pela APS.

Estudo sobre abandono do tratamento no PNCT verificou que a maioria dos fumantes realizou entre uma e três tentativas prévias de cessação ${ }^{(16)}$. Essa informação tem relevância para nortear condutas e intervenções, no sentido de evitar adotar práticas já realizadas e que não obtiveram sucesso.

A partir do estudo realizado, observou-se a escassez de trabalhos que buscam entender as dificuldades de adesão aos grupos de cessação tabágica, o que dificultou uma discussão mais aprofundada sobre o tema. Destaca-se, ainda, como limitação da pesquisa, a incompatibilidade entre os dados cadastrais do usuário tabagista e a realidade observada, como números de telefone desatualizados ou ausentes, endereços incorretos e indivíduos não mais tabagistas ainda registrados como tal.

\section{CONCLUSÃO}

Constatou-se que a existência de um programa específico - o PNCT - e suas próprias iniciativas locais, a exemplo do grupo de cessação tabágica ofertado pela APS, ainda é pouco difundida, o que parece propiciar abaixa adesão a eles.

Dessa forma, recomenda-se a valorização da busca ativa e o desenvolvimento de novas estratégias de recrutamento dos indivíduos na comunidade, considerando os meios de locomoção e horários de disponibilidade, além de abordagem dinâmica a ser adotada nos grupos que atenda à real necessidade dos participantes. Abordagens com alvo nos fatores desencadeantes do desejo de fumar - ansiedade, depressão, estresse, hábitos alimentares - poderiam ser incorporadas aos grupos, bem como momentos de atenção individual. Acredita-se que essas medidas possam contribuir para o sucesso na participação do programa e consequentemente na cessação do hábito de fumar.

Destaca-se, ainda, a importância da qualificação profissional para garantir que as diretrizes preconizadas pelo PNCT sejam aplicadas e o melhor plano terapêutico seja ofertado ao usuário, assegurando a acessibilidade.

A dificuldade em encontrar trabalhos sobre adesão e sucesso dos grupos de cessação evidencia a necessidade de novos estudos que busquem entender melhor as necessidades dos tabagistas e suas dificuldades. A compreensão dos fatores que influenciam a dinâmica das ações de cessação até a concretização de seus objetivos mostra-se fundamental para o cumprimento das diretrizes nacionais na esfera local.

\section{REFERÊNCIAS}

1. World Health Organization (WHO). Tobacco. Fact sheet №339. [Internet] Geneva: WHO; 2015 [acesso em 17 set 2017]. Disponível: http://www.who.int/mediacentre/factsheets/fs339/en/.

2. World Health Organization (WHO). Global status report on non communicable diseases 2010. [Internet] Geneva: WHO; 2011 [acesso em 25 mar 2018]. Disponível: www.who.int/nmh/publications/ 
ncd_report_full_en.pdf.

3. World Health Organization (WHO). Who global report mortality attributable to tobacco. [Internet] Geneva: WHO; 2011 [acesso em 25 mar 2018]. Disponível: http://whqlibdoc.who.int/publications/2012/9789241564434_eng.pdf.

4. Global Burden of Disease. Global, regional, and national comparative risk assessment of 79 behavioural, environmental and occupational, and metabolic risks or clusters of risks, 1990-2015: a systematic analysis for the Global Burden of Disease Study 2015. Lancet. [Internet] 2016;388 [acesso em 25 mar 2018]. Disponível: https://www.ncbi.nlm.nih.gov/pmc/articles/PMC5388856/.

5. Pinto M, Bardach A, Palacios A, Biz AN, Alcaraz A, Rodri'guez B, et al. Carga de doença atribuível ao uso do tabaco no Brasil e potencial impacto do aumento de preços por meio de impostos. [Documento técnico] IECS 21. [acesso em 25 mar 2018]. Disponível: http://www.iecs.org.ar/wp-content/uploads/ Reporte-completo.pdf.

6. Campos PCM, Gomide M. O Programa Nacional de Controle do Tabagismo (PNCT) na perspectiva social: a análise de redes, capital e apoio social. Cad. saúde colet. [Internet] 2015;23(4) [acesso em 20 set 2017]. Disponível: http://dx.doi.org/10.1590/1414-462X201500040241.

7. Instituto Nacional de Câncer (INCA). Programa Nacional de Controle do Tabagismo. [Internet] Rio de Janeiro: INCA; 2014 [acesso em 18 set 2018]. Disponível: http://www2.inca.gov.br/wps/wcm/connect/ acoes_programas/site/home/nobrasil/programa-nacional-controle-tabagismo/programa-nacional.

8. Ministério da Saúde (BR). Portaria no 571, de 5 de abril de 2013. Atualiza as diretrizes de cuidado à pessoa tabagista no âmbito da Rede de Atenção à Saúde das Pessoas com Doenças Crônicas do Sistema Único de Saúde (SUS) e dá outras providências. Diário Oficial da União, 5 abr 2013.

9. Vaz-Carneiro A, Costa J. Análise da Revisão Cochrane: Incentivos na Cessação Tabágica. Cochrane DatabaseSyst. Revista Científica da Ordem dos Médicos. [Internet] 2016;9(1) [acesso em 22 set 2017]. Disponível: https://www.actamedicaportuguesa.com/revista/index.php/amp/article/viewFile/7363/4568.

10. Lopes FM, Peuker ACWB, Rech BE, Gonçalves R; Bizarro L. Desenvolvimento, divulgação, adesão e eficácia de um Programa de Cessação do Tabagismo oferecido e uma universidade pública. Cadernos Brasileiros de Terapia Ocupacional. [Internet] 2014;22 (n.esp) [acesso em 22 set 2017]. Disponível: https://doi.org/10.4322/cto.2014.025.

11. Portes LH, Campos SEM, Teixeira MTB, Caetano R, Ribeiro LC. Ações voltadas para o tabagismo: análise de sua implementação na Atenção Primária à Saúde. Ciênc. saúde coletiva. [Internet] 2014;19(2) [acesso em 21 set 2017]. Disponível: http://dx.doi.org/10.1590/1413-81232014192.04702013.

12. Pantani D, Pinsky I, Monteiro A. Publicidade de tabaco no ponto de venda. Instituto Nacional de Ciência e Tecnologia para Políticas Públicas do Álcool e Outras Drogas. São Paulo - SP; 2011 [acesso em 23 set 2017]. Disponível: https://www.uniad.org.br/images/stories/arquivos/PUBLICIDADE\%20 DE\%20TABACO\%20para\%20dowload.pdf.

13. da Silva ST, Martins MC, de Faria FR, Cotta RMM. Combate ao Tabagismo no Brasil: a importância estratégica das ações governamentais. Ciênc. saúde coletiva. [Internet] 2014;19(2) [acesso em 21 set 2017]. Disponível: http://dx.doi.org/10.1590/1413-81232014192.19802012.

14. Almeida GBS, Rodrigues JP, Freire MR. Acessibilidade dos usuários ao Programa de Controle do Tabagismo. Revista HU. [Internet] 2013;39 (3 e 4) [acesso em 21 set 2017]. Disponível: https://hurevista. ufjf.emnuvens.com.br/hurevista/article/viewFile/2154/767.

15. Reichert J, de Araújo AJ, Gonçalves CMC, Godoy I, Chatkin JM, Sales PU, et al. Smoking cessation guidelines - 2008. J. bras. pneumol. [Internet] 2008;34(10) [acesso em 25 mar 2018]. Disponível: http:// dx.doi.org/10.1590/S1806-37132008001000014.

16. Meier DAP, Vannuchi MTO, Secco IAO. Abandono do tratamento do tabagismo em programa de município do Paraná. Revista de Saúde Pública do Paraná. [Internet] 2011;13(1) [acesso em 22 set 2017]. Disponível: http://www.uel.br/revistas/uel/index.php/espacoparasaude/article/view/10020/9349. 
17. Lopes FM, Peuker AC, Bizarro L. Aplicação de um programa de Cessação do tabagismo com rodoviários. Psicol. cienc. prof. [Internet] 2013;33(2) [acesso em 22 set 2017]. Disponível: http://dx.doi. org/10.1590/S1414-98932013000200017.

18. de Souza PS, Moreira J, Victor EG, Ceretta LB, Tuon L. Educação em saúde nos grupos de controle ao tabagismo no município de Criciúma: a prática do fisioterapeuta. Revista Saúde Publica. [Internet] 2013;6(3) [acesso em 22 set 2017]. Disponível em: http://cienciaparaeducacao.org/eng/publicacao/ souza-p-s-moreira-j-victor-e-g-ceretta-luciane-b-tuon-I-educacao-em-saude-nos-grupos-de-controleao-tabagismo-no-municipio-de-criciuma-a-pratica-do-fisioterapeuta-revista-de-sa/.

19. Formagini TDB, Machado NM, Richter KP, Ronzani TM. Intervenções de Cessação de Tabagismo em Fumantes Leves : Uma Revisão Sistemática. Psicologia em Estudo. [Internet] 2015;20(2) [acesso em 23 set 2017]. Disponível: http://dx.doi.org/10.4025/psicolestud.v20i2.24908.

20. Duarte MLC, de Campos LMP, Monteiro LR, de Oliveira MC. O grupo de tabagismo em um ambulatório de saúde mental no rio grande do sul. Rev. Contexto Saúde. [Internet] 2012;14(27) [acesso em 23 de setembro de 2017]. Disponível: http://hdl.handle.net/10183/116240.

21. Brasil. Instituto Nacional de Câncer José Alencar Gomes da Silva; Organização Pan-Americana da Saúde. Pesquisa especial de tabagismo - PETab: relatório Brasil. Rio de Janeiro: INCA, 2011.

22. Berto SJP, Carvalhaes MABL, Moura EC. Tabagismo associado a outros fatores comportamentais de risco de doenças e agravos crônicos não transmissíveis. Cad. Saúde Pública. [Internet] 2010;26(8) [acesso em 08 fev 2018]. Disponível: https://www.scielosp.org/pdf/csp/2010.v26n8/1573-1582.

23. dos Reis RCM, Fortes RC. Fatores associados a não cessação do tabagismo em participantes do grupo de terapia de um centro de saúde do Distrito Federal. Revista Faccesa. [Internet] 2012;1(1) [acesso em 23 set 2017]. Disponível em: http://revistafacesa.senaaires.com.br/index.php/revisa/article/view/7. 